\title{
Body Diffusion Kurtosis Imaging: Basic Principles, Applications, and Considerations for Clinical Practice
}

\author{
Andrew B. Rosenkrantz, MD, ${ }^{1 *}$ Anwar R. Padhani, MB, BS, ${ }^{2}$ \\ Thomas L. Chenevert, PhD, ${ }^{3}$ Dow-Mu Koh, MD, MRCP, FRCR, ${ }^{4}$ \\ Frederik De Keyzer, MSc, ${ }^{5}$ Bachir Taouli, MD, ${ }^{6}$ and Denis Le Bihan, MD, PhD ${ }^{7}$
}

\begin{abstract}
Technologic advances enable performance of diffusion-weighted imaging (DWI) at ultrahigh b-values, where standard monoexponential model analysis may not apply. Rather, non-Gaussian water diffusion properties emerge, which in cellular tissues are, in part, influenced by the intracellular environment that is not well evaluated by conventional DWI. The novel technique, diffusion kurtosis imaging (DKI), enables characterization of non-Gaussian water diffusion behavior. More advanced mathematical curve fitting of the signal intensity decay curve using the DKI model provides an additional parameter $\mathrm{K}_{\mathrm{app}}$ that presumably reflects heterogeneity and irregularity of cellular microstructure, as well as the amount of interfaces within cellular tissues. Although largely applied for neural applications over the past decade, a small number of studies have recently explored DKI outside the brain. The most investigated organ is the prostate, with preliminary studies suggesting improved tumor detection and grading using DKI. Although still largely in the research phase, DKI is being explored in wider clinical settings. When assessing extracranial applications of DKI, careful attention to details with which body radiologists may currently be unfamiliar is important to ensure reliable results. Accordingly, a robust understanding of DKI is necessary for radiologists to better understand the meaning of DKI-derived metrics in the context of different tumors and how these metrics vary between tumor types and in response to treatment. In this review, we outline DKI principles, propose biostructural basis for observations, provide a comparison with standard monoexponential fitting and the apparent diffusion coefficient, report on extracranial clinical investigations to date, and recommend technical considerations for implementation in body imaging.
\end{abstract}

J. MAGN. RESON. IMAGING 2015;42:1190-1202.

$\mathrm{D}$ iffusion-Weighted Imaging (DWI) has become established as a powerful magnetic resonance imaging (MRI) technique for evaluating pathology throughout the body and is now routinely incorporated into many body MRI protocols, mainly in oncology. ${ }^{1,2}$ DWI is performed by serially imaging the same tissue while varying the degree of water diffusion sensitization. The imaging gradient strength, direction, and temporal profile affect sensitivity to diffusion and are commonly reduced to a single simplified parameter referred to as the b-value [unit: $s / \mathrm{mm}^{2}$ ]. The images obtained at different $b$-values are subsequently used for computing a parametric map that allows quantitative assessment of the tissue's water diffusion behavior. In clinical body imaging, DWI is typically performed using b-values up to $800-1000 \mathrm{~s} / \mathrm{mm}^{2}$, and subsequent quantification is typically performed using a monoexponential fit. ${ }^{3}$ For simplicity, this model assumes Gaussian (free) diffusion behavior, in which free tissue diffusion would yield a normal distribution of tissue diffusivities. This Gaussian diffusion behavior would result in linear decay of the natural logarithm of the DWI signal intensity (SI) as the b-value increases, the slope of which is referred to as the apparent

View this article online at wileyonlinelibrary.com. DOI: 10.1002/jmri.24985

Received Apr 27, 2015, Accepted for publication Jun 10, 2015.

*Address reprint requests to: A.B.R., Department of Radiology, Center for Biomedical Imaging, NYU School of Medicine, NYU Langone Medical Center, 660 First Ave., 3rd Floor, New York, NY 10016. E-mail: Andrew.Rosenkrantz@nyumc.org

From the ${ }^{1}$ Department of Radiology, Center for Biomedical Imaging, NYU School of Medicine, NYU Langone Medical Center, New York, New York, USA;

${ }^{2}$ Paul Strickland Scanner Centre, Mount Vernon Cancer Centre, Northwood, Middlesex, UK; ${ }^{3}$ University of Michigan Health System, Department of

Radiology - MRI, Ann Arbor, Michigan, USA; ${ }^{4}$ Department of Radiology, Royal Marsden NHS Foundation Trust, Sutton, UK; ${ }^{5}$ Department of Radiology,

University Hospitals Leuven, Leuven, Belgium; ${ }^{6}$ Department of Radiology, Translational and Molecular Imaging Institute, Icahn School of Medicine at Mount Sinai, New York, New York, USA; and ${ }^{7}$ NeuroSpin, I²BM/DSV/CEA, Gif-sur-Yvette, France. 
diffusion coefficient (ADC; unit: $\times 10^{-3} \mathrm{~mm}^{2} / \mathrm{s}, \mu \mathrm{m}^{2} / \mathrm{msec}$, or $\left.\times 10^{3} \mu \mathrm{m}^{2} / \mathrm{s}\right)$ to emphasize that it is not the real diffusion coefficient. ${ }^{3}$ ADC is derived from the equation: $S_{i}=S_{0} * e^{\left(-b_{i} * A D C\right)}$, in which $S_{\mathrm{i}}$ is the $\mathrm{SI}$ at b-value $\mathrm{b}_{\mathrm{i}}$, and $S_{0}$ represents the estimated $S I$ at a b-value of 0 in the absence of any noise contribution.

Historically, MRI scanners were limited in their abilities to obtain images at b-values greater than $1000 \mathrm{~s} / \mathrm{mm}^{2}$ due to insufficient signal-to-noise ratio (SNR) and increased anatomic distortions resulting from field inhomogeneity and induced eddy currents. However, improvements in hardware and software within modern MRI systems now enable the acquisition of ultrahigh b-value images $\left(>1000 \mathrm{~s} / \mathrm{mm}^{2}\right)$, which when acquired with good image SNR have been shown to enhance clinical disease assessment for prostate cancer detection and glioma characterization. ${ }^{4,5}$ Acquiring DWI at ultrahigh b-values often reveals the presence of non-Gaussian diffusion effects, thus requiring a more sophisticated model for analysis. One such model is termed diffusion kurtosis imaging (DKI), which potentially provides more information on tissue structure than does standard monoexponential analysis for b-values less than $1000 \mathrm{~s} /$ $\mathrm{mm}^{2}$. DKI was first described by studies in $2004^{6}$ and $2005^{7}$ and initially was applied exclusively for brain imaging. ${ }^{5,8,9}$ However, in recent years studies have shown the feasibility of applying DKI at multiple extracranial sites. $^{10-13}$ Therefore, body radiologists may benefit through a better understanding of the major concepts of DKI. In this article, we review the basic principles of DKI, proposed biologic correlates, technical considerations to facilitate clinical translation, and potential applications outside the brain reported within the recent peer-reviewed literature.

\section{DKI: Underlying Model}

Past studies have reported on the potential value of DWI using b-values over $1000 \mathrm{~s} / \mathrm{mm}^{2}$ in oncologic applications, almost all showing improved tumor conspicuity against a fading background signal intensity on the ultrahigh b-values images. ${ }^{14-16}$ However, these studies have commonly postprocessed the image sets using a standard monoexponential fit to obtain ADC maps. ${ }^{17-19}$ While this approach may appear straightforward, there is an assumption of monoexponential behavior of water diffusivity. The monoexponential model, which applies a linear fit to the natural logarithm of the SI, is reasonable at b-values up to $\sim 600$ $1000 \mathrm{~s} / \mathrm{mm}^{2}$, depending on the given tissue. However, as the b-value increases, the logarithmic SI decay plot no longer maintains a linear shape, but rather exhibits a distinct curvature with a positive deviation from the plot of the monoexponential model ${ }^{7}$ (Fig. 1). This curvature indicates the presence of water diffusion behaviors away from Gaussian predictions, and accordingly, that alternate models should be applied when observed. ${ }^{7}$

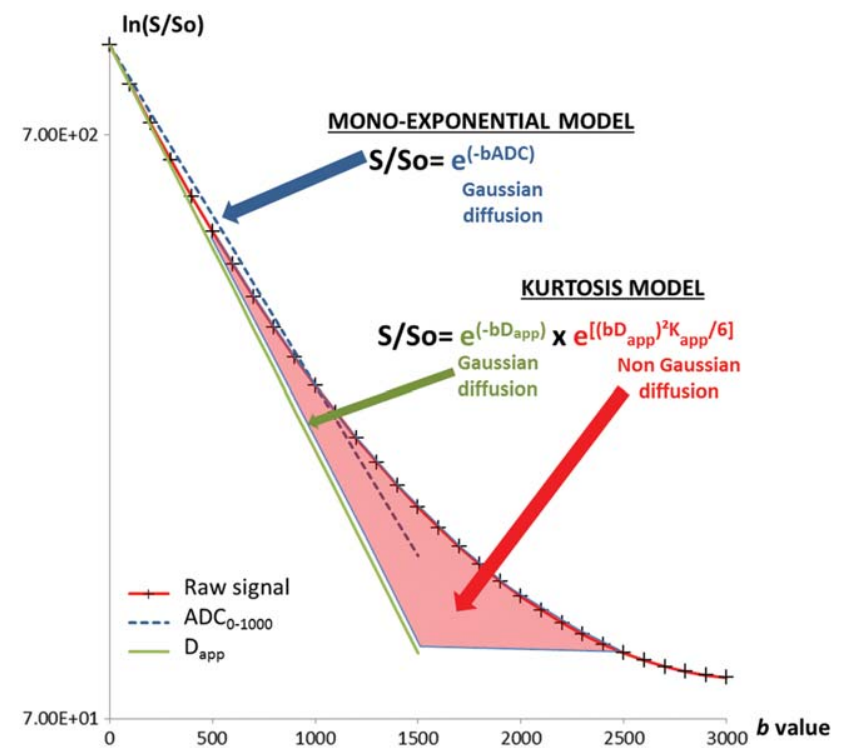

FIGURE 1: Comparison of diffusion signal intensity decay plots for monoexponential model, reflecting solely Gaussian diffusion, and kurtosis model, reflecting both Gaussian and nonGaussian diffusion components.

DKI analyses non-Gaussian water diffusivity using a polynomial model according to the following equation: $S_{i}=S_{0} * e^{\left(-b_{i} * D a p p+\frac{1}{6} * b_{i}^{2} * D_{a p p}^{2} * K_{a p p}\right)}$. In comparison with the monoexponential equation, this equation yields two variables $\left(D_{\text {app }}\right.$ and $K_{\text {app }}$ ) when $S_{0}$ is known. The parameter $\mathrm{K}_{\mathrm{app}}$ represents the apparent diffusional kurtosis (unitless), and $D_{\text {app }}$ is the diffusion coefficient (unit: $\times 10^{-3} \mathrm{~mm}^{2} / \mathrm{s}$, $\mu \mathrm{m}^{2} / \mathrm{msec}$, or $\times 10^{3} \mu \mathrm{m}^{2} / \mathrm{s}$ ) that is corrected to account for the observed non-Gaussian behavior. For histogram descriptions, a distribution with greater kurtosis has a more peaked distribution in comparison with a normal distribution (Fig. 2). Accordingly, $K_{a p p}$ reflects the more peaked distribution of tissue diffusivities that occurs in the setting of nonGaussian diffusion behavior. $\mathrm{K}_{\mathrm{app}}$ is determined by the SI decay curvature away from the plot that would be predicted by a monoexponential fit, ${ }^{7}$ whereas $\mathrm{D}_{\text {app }}$ is determined by the slope of the SI decay plot as b approaches 0 .

Not only does the DKI model potentially better reflect water diffusivity in tissues at ultrahigh b-values, it also provides an additional parameter $K_{\text {app }}$ that contains specific information on the non-Gaussian diffusion behavior. Thus, the DKI model provides radiologists with an opportunity to potentially gain further insights into tissue characteristics than are obtained through standard DWI. Other models for fitting extended diffusion data are described, including the biexponential model that separates the water diffusivity into two components (fast and slow, with their respective relative volumes and diffusivities), as well as the stretchedexponential model that views water diffusion as comprising multiple Gaussian compartments with a wide distribution of diffusivities. $^{20,21}$ However, given the noise inherent in 


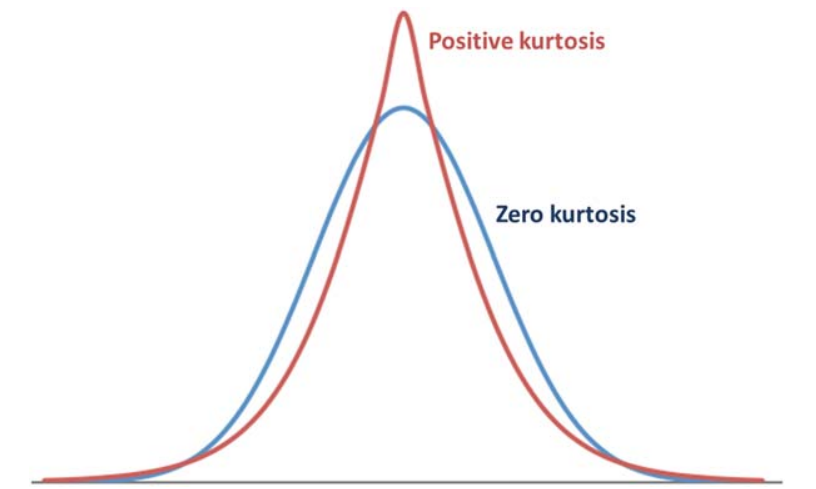

FIGURE 2: Comparison of Gaussian distribution (blue curve) with kurtosis of 0 and non-Gaussian distribution (red curve) with greater peak and consequent positive kurtosis.

clinical datasets, the DKI model is potentially the most robust, as it has only two unknown parameters $\left(D_{\text {app }}\right.$ and $\mathrm{K}_{\mathrm{app}}$ ) that need to be estimated when $S_{0}$ is known or can be derived. ${ }^{3,12}$

\section{Biostructural Basis}

$\mathrm{K}_{\text {app }}$ is a phenomenological parameter, ${ }^{22}$ supported by observations of it providing excellent mathematical fitting of the SI decay plot at very high b-values (although lower than 2500-3000 s/ $\left.\mathrm{mm}^{2}\right) .^{3}$ It has no direct biophysical basis and in this sense it is similar to ADC, which also represents an "apparent" parameter that has only indirect biophysical correlates. Nonetheless, the underlying basis of DKI metrics have been hypothesized. ${ }^{3}$ Tissue ADC measurements at bvalues less than $1000 \mathrm{~s} / \mathrm{mm}^{2}$ have been ascribed as a measure largely of the extracellular space, ${ }^{3,22}$ reflecting the extent to which water molecules are hindered outside of cells and so are influenced by tissue architectural properties that include vessels, ducts, and extracellular space tortuosity. Thus, cellular arrangements, cell size distributions, cellular density, extracellular space viscosity, glandular structures, and integrity of membranes all have the potential to modulate extracellular space water motion. Accordingly, reduced ADC has often been attributed to increasing cellular density, given the effect of hydrophobic cell membranes in limiting extracellular water motion. ${ }^{3}$ However, the degree of water hindrance scaling with respect to cellularity is dependent on a uniform distribution of cell sizes, and the inverse relationship between cellularity and ADC does not apply to all tissues. For example, in bone marrow the presence of smaller sized hematopoietic cells intermixed with larger fat cells initially increases ADC with increasing cellularity. ${ }^{23,24}$ Radiologists should also note that ADC correlates are not specific for the effects of cellularity on water motion, which can also be hindered simply by the presence of a greater concentration of macromolecules and increased viscosity. ${ }^{7}$

In comparison, $\mathrm{K}_{\mathrm{app}}$ of tissues has been hypothesized at least in part to represent the direct interaction of water molecules with cell membranes and intracellular compounds, ${ }^{3,7,22}$ although is also influenced by other extracellular factors that are difficult to separate. Le Bihan ${ }^{25}$ suggests that water is not a homogeneous substance at the nanoscopic level, given the polar nature of the water molecule, which results in the formation of network clusters with differing degrees of hydrogen bonding and tetrahedrality. Furthermore, liquid water may form $3 \mathrm{D}$ arrays in the presence of interfaces with charged materials such as polarized cell or organelle membranes or protein molecules. Extensive selfassociations of water within charged environments result in organization of water molecules into layers with reduced diffusivity. In cells, proteins have an especially profound effect on water, given their presence of a charge that results in protein-water adsorption. These factors, taken together, may result in the marked hindrance of water motion, contributing to non-Gaussian diffusion observations. Increased kurtosis is suggested to occur in the setting of more irregular and heterogeneous environments with many or large interfaces, ${ }^{7,26}$ including the increased nuclear-cytoplasmic ratio of tumor cells. Kurtosis measurements potentially have greater specificity to reveal water interactions within cell and tissue components. ${ }^{7}$ A prior study using a synthetic tumor cell environment that observed diffusion signal intensities to be sensitive to intracellular architecture but not to overall cell density supports the relationship between ultrahigh b-value DWI and intracellular water behavior. ${ }^{27}$ Studies that observed associations between DKI metrics and microstructural properties of prostate cancer support this framework as well. ${ }^{28,29}$ Nonetheless, work remains to be done to fully understand the mechanisms underlying non-Gaussian diffusion, including other noncell-based influences and the precise biostructural significance of $K_{\text {app }}$.

\section{Acquisition and Postprocessing Considerations}

In this section, we provide technical recommendations to guide body radiologists in implementing DKI in routine clinical practice. Table 1 provides general considerations, while Table 2 provides the specific acquisition parameters for a representative sample protocol for performing DKI of the prostate.

\section{Image Acquisition}

DKI is acquired using a standard DWI sequence available on current-generation scanners, albeit incorporating ultrahigh b-values. Whereas standard DWI requires acquisition of only two b-values for the calculation of ADC, DKI requires acquisition of at least three distinct b-values given an additional unknown variable $\left(\mathrm{K}_{\mathrm{app}}\right)$ within the formula. ${ }^{3}$ In practice, we suggest acquiring more than three b-values, including at least two b-values both above and below $1000 \mathrm{~s} / \mathrm{mm}^{2}$, to facilitate the successful capture of the non- 
Rosenkrantz et al.: Body Diffusion Kurtosis Imaging

\section{TABLE 1. Technical Considerations for Body Applications of DKI}

\begin{tabular}{|c|c|}
\hline Factor & Suggestion \\
\hline $\begin{array}{l}\text { Underlying pulse } \\
\text { sequence }\end{array}$ & $\begin{array}{l}\text { Standard diffusion-weighted pulse sequence (spin-echo echo-planar imaging) with mini- } \\
\text { mum possible TE }\end{array}$ \\
\hline Number of b-values & $\begin{array}{l}\text { At least } 3 \text { b-values needed; at least } 2 \text { b-values both above and below b1000 s/mm may } \\
\text { facilitate robust estimates of } D_{\text {app }} \text { and } K_{\text {app }}\end{array}$ \\
\hline Minimum b-value & $\begin{array}{l}\text { Although b0 is acceptable, b-values } \geq 200 \mathrm{~s} / \mathrm{mm}^{2} \text { are preferred to reduce perfusion effects. } \\
\text { b-values in the } 0-200 \mathrm{~s} / \mathrm{mm}^{2} \text { range are useful only when also estimating perfusion effects } \\
\text { (IVIM DWI) }\end{array}$ \\
\hline Maximum b-value & At least $1500 \mathrm{~s} / \mathrm{mm}^{2}$; avoid excessively high b-values (over $\sim 3000 \mathrm{~s} / \mathrm{mm}^{2}$ ) \\
\hline Number of directions & Three directions sufficient; acquiring full tensor not necessary \\
\hline SNR considerations & $\begin{array}{l}\text { Sufficient SNR critical; consider increasing number of averages, adjusting image geometry, } \\
\text { and employing mathematical noise compensation algorithms }\end{array}$ \\
\hline Postprocessing & Dedicated postprocessing software that generates, at a minimum, $K_{\text {app }}$ and $D_{a p p}$ maps \\
\hline
\end{tabular}

Gaussian behavior. If the maximal b-value is not high enough, then the deviation of the curvature of the SI decay plot away from Gaussian will not be captured, and the ability of the sequence to measure non-Gaussian diffusion behavior decreases substantially. ${ }^{33}$ In the brain, DKI is performed using a maximal $b$-value in the range of 2000$3000 \mathrm{~s} / \mathrm{mm}^{2}{ }^{2,34}$ However, excessively high b-values over $3000 \mathrm{~s} / \mathrm{mm}^{2}$ lead to SI measurements that violate assumptions of the DKI model and are therefore discour-

\section{TABLE 2. Acquisition Parameters for Sample DKI Protocol of the Prostate}

\begin{tabular}{ll} 
Parameter & Sample value \\
\hline Pulse sequence & $\begin{array}{l}\text { Free-breathing spin-echo } \\
\text { fat-suppressed echo-planar } \\
\text { imaging }\end{array}$ \\
\hline TR & $3000 \mathrm{msec}$ \\
\hline TE & $70 \mathrm{msec}$ \\
\hline FOV & $260 \mathrm{~mm} \times 80 \%$ rectangular FOV \\
\hline $\begin{array}{l}\text { Acquisition matrix } \\
\text { Slice thickness }\end{array}$ & $100 \times 100$ (before interpolation) \\
\hline $\begin{array}{l}\text { Averages } \\
\begin{array}{l}\text { Parallel imaging } \\
\text { reduction factor }\end{array}\end{array}$ & 20 (for the largest b-values) \\
\hline $\begin{array}{l}\text { Receiver } \\
\text { bandwidth }\end{array}$ & $2000 \mathrm{~Hz} /$ voxel \\
$\begin{array}{l}\text { b-values } \\
\text { (sec/mm }\end{array}{ }^{2}$ ) & $200,500,1000,1500,2000$ \\
\hline $\begin{array}{l}\text { Diffusion } \\
\text { directions }\end{array}$ & Three-directional trace \\
\hline
\end{tabular}

aged. $^{7,12,22,26,34}$ Q-space imaging is a related diffusion technique that, although employing much larger b-values than those used for DKI, also provides estimates of K., ${ }^{6,26,35}$ This more advanced technique has greater hardware, acquisition time, and postprocessing requirements and is beyond the scope of this article. ${ }^{7,26}$

Compared with the brain, tissues in the body generally exhibit faster signal decays at increasing b-values, as well as faster $T_{2}$ decays. ${ }^{36}$ Moreover, larger-sized torso coils have less ideal receptivity and geometry in comparison with head coils. Therefore, the optimal choice of maximal b-value for DKI in body applications is lower than in the brain. Indeed, non-Gaussian behavior of the SI decay plot can be successfully captured at a maximal b-value of $\sim 1500-2000 \mathrm{~s} / \mathrm{mm}^{2}$ in body imaging. ${ }^{11,37,38}$ On this basis, we suggest that a maximal b-value in this range may be appropriate for DKI body applications.

Selection of the minimal b-value for DKI should take into account sensitivity to intravoxel incoherent motion (IVIM) effects that are visible at very low b-values (typically lower than $400 \mathrm{~s} / \mathrm{mm}^{2}$ ). These b-values are theoretically far from the high b-values used for DKI, such that the acquisition of multiple, very low b-value images, although standard for IVIM assessment, is, in principle, not needed when performing DKI. ${ }^{39}$ While a minimal b-value of 0 is acceptable, a higher minimal b-value of $\geq 200 \mathrm{~s} / \mathrm{mm}^{2}$ may be useful to mitigate the effect of capillary perfusion on SI measurements occurring at lower b-values. ${ }^{26,36}$ Nonetheless, because signals are noisy, curve-fitting algorithms may incorrectly assign part of the signal curvature to either IVIM or kurtosis effects, thus misestimating model parameters. ${ }^{12}$ Alternatively, obtaining a few lower b-value images (b-values less than $1000 \mathrm{~s} / \mathrm{mm}^{2}$ ), in addition to the high b-values that are required for DKI, may be considered, with recent work 
demonstrating the ability to perform a comprehensive analysis incorporating both IVIM and DKI effects. ${ }^{12,40}$

In order for the calculated DKI metrics to be accurate, it is critical for the high b-value images to exhibit sufficient SNR. ${ }^{34}$ Otherwise, the SI decay plot approaches the "noise floor," which in turn leads to an artifactual curvature of the SI decay plot and yields biased estimates of $\mathrm{K}_{\mathrm{app}} \cdot{ }^{12,41}$ The rate at which signal approaches the noise floor depends on diffusion properties of the tissue itself. That is, it is conceivable to have adequate SNR for $K_{\text {app }}$ estimation in one region (eg, high-grade prostate tumor) and inadequate SNR, leading to biased $\mathrm{K}_{\mathrm{app}}$ estimation in adjacent tissue (eg, normal prostate parenchyma). Consistently obtaining sufficient SNR at high b-values is particularly challenging in body imaging, given not only faster signal decays, but also the tendency to use faster acquisitions to compensate for respiration and other sources of motion. Therefore, it may be necessary to reduce the spatial resolution or increase the number of signal averages to maintain SNR. When allocating the number of averages, fewer averages may be required for the lower b-values, thereby enabling more averages to be allocated to the high b-value images, thus increasing their SNR. Given the pronounced SNR demands of DKI, optimized sequence parameters are likely to be different from that of conventional DWI in the same tissue.

As breath-hold imaging is associated with significantly reduced SNR for DWI, ${ }^{42}$ either respiratory-triggered or free-breathing acquisitions are needed. Using the minimal possible echo time also helps raise SNR, which is facilitated at ultrahigh b-values through high-performance gradient systems. Use of a 3T system, when available, substantially improves SNR as well. Even with these considerations, SNR may still be insufficient. Post-hoc corrections of "noise floor" effects in the signal remain challenging, although some simple techniques have been proposed for clinical practice. ${ }^{12,41}$ Such techniques show an improved reliability of DKI measurements and may be an important step towards achieving routine clinical applicability of DKI in the body. ${ }^{12}$ When incorporating noise compensation, an SNR of at least 2 on the high b-value images has been suggested as a minimum threshold for allowing for a reasonable estimate of $\mathrm{K}_{\mathrm{app}}{ }^{41}$ However, even higher SNR is preferred, to the extent achievable.

As already noted, DKI requires a minimum of only three b-values. In fact, it may be advantageous to avoid acquiring an excessive number of b-values. ${ }^{26} \mathrm{~A}$ very large number of $b$-values increases the overall scan time, both increasing the likelihood of motion artifact and hindering incorporation of DKI into clinical protocols. On the other hand, a limited number of b-values in both high (5001000) and ultrahigh (1500-2000) b-value ranges may be useful for successfully capturing the monoexponential and non-Gaussian components, respectively, of the SI decay curve. Therefore, the optimal number of b-values to obtain, as well as their distribution across the acquired range of bvalues, cannot be strictly prescribed and will depend on the clinical application, desire to obtain simultaneous IVIM and/or ADC estimates, expected SNR, likelihood of motion artifacts, scanner capability, and available imaging time.

Some studies report performing kurtosis assessment of the full multidirectional diffusion tensor, described as diffusion kurtosis tensor imaging (DKTI). ${ }^{43,44}$ This approach acquires diffusion images in at least 15 different directions in order to assess the anisotropic directionality of diffusion and kurtosis behavior. ${ }^{7}$ Similar to the above consideration of the number of b-values, acquisition of this many directions is not necessary for oncologic DKI. Rather, DKI can be performed based on a directionless "trace" of the diffusion tensor, which requires acquisition of only three directions. ${ }^{7}$ Given the prohibitive scan time of the 15 directions needed to perform DKTI, DKI of the body has been performed overwhelmingly in a directionless fashion. This scheme highlights that DKI does not require tissue anisotropy and indeed provides markedly distinct information compared with DTI-derived metrics such as fractional anisotropy. ${ }^{7}$

Finally, although not a parameter routinely manipulated by radiologists, the diffusion time, DT, indicating the time between onset of the two lobes of the diffusionencoding gradient, also impacts kurtosis estimates, given the greater physical diffusion distances probed with increasing DT. A short DT is advised in order to be sensitive to obstacles to non-Gaussian water diffusion that occur at short length scales and that influence $K_{a p p}$, as well as to mitigate water exchange between tissue compartments that occurs at longer DT. ${ }^{40} \mathrm{~K}_{\text {app }}$ increases as DT increases from a short DT, reaching a peak value at an intermediate DT, and subsequently decreases with further increases in DT, ultimately approaching zero. ${ }^{26,40}$ In comparison, $\mathrm{D}_{\text {app }}$ is relatively less sensitive to DT length. ${ }^{40}$ Shorter DT may be achieved by minimizing TE times, which is facilitated through use of monopolar diffusion-encoding schemes ${ }^{45,46}$ and stronger gradient rise times.

\section{Image Postprocessing}

While DKI is acquired using standard DWI sequences, current clinical MR systems do not routinely provide in-line DKI postprocessing options. Therefore, separate postprocessing software is needed, which is becoming increasingly available. As a minimum, DKI analysis should provide at least two parametric maps corresponding to $\mathrm{D}_{\text {app }}$ and $\mathrm{K}_{\text {app }}$, while $\mathrm{b}_{0}$ and user-defined computed b-value images can also be generated. $\mathrm{D}_{\text {app }}$ is analogous to the metric ADC that is familiar to radiologists, being reduced in the setting of impeded diffusion. While numerous factors influence these metrics, $D_{\text {app }}$ is presumed to be the more accurate measure 


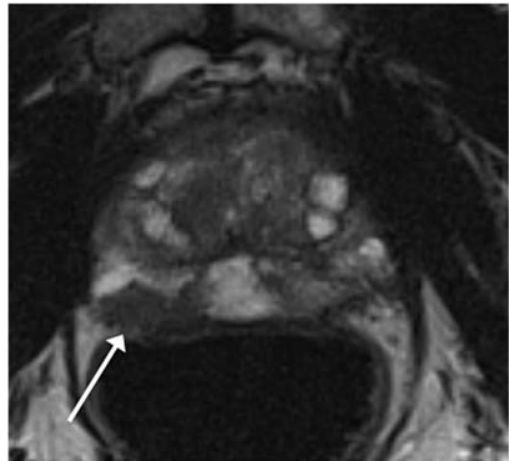

(a)

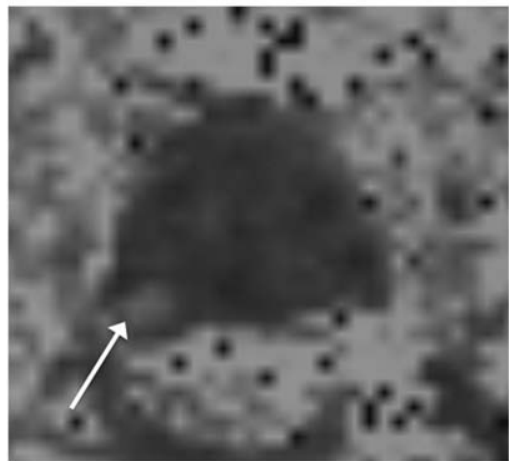

(d)

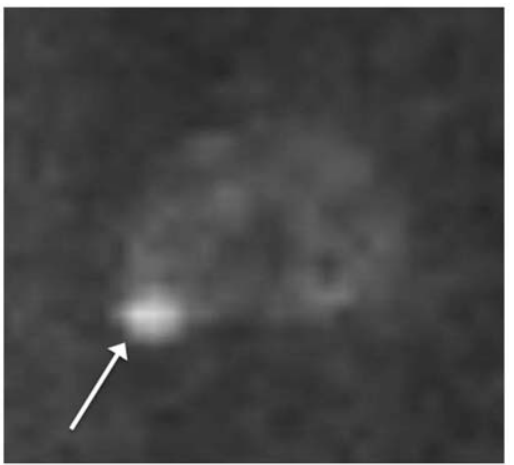

(b)

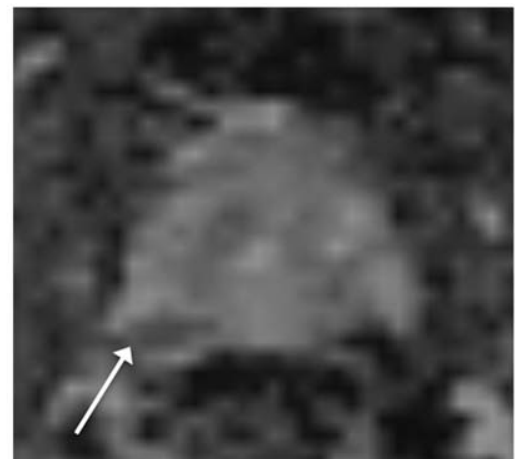

(c)

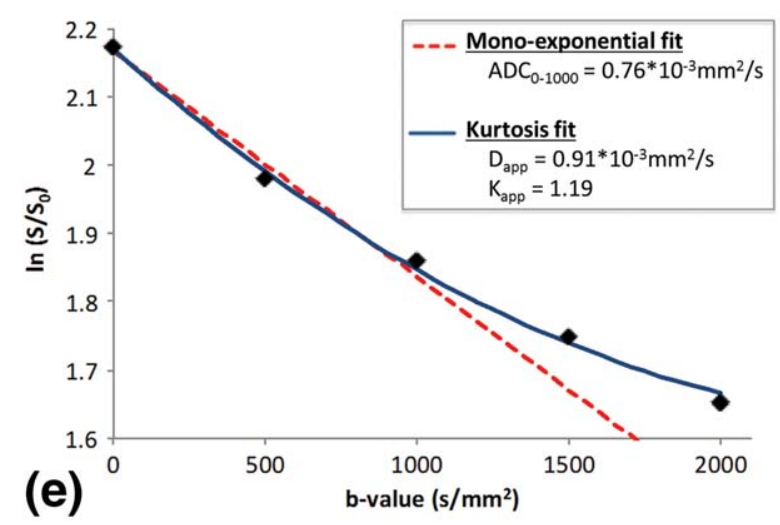

FIGURE 3: A 68-year-old man with Gleason $4+3$ prostate cancer in right peripheral zone. A: Axial $T_{2}$-weighted image shows decreased $T_{2}$ signal in lesion (arrow). B: Diffusion-weighted image with ultrahigh b-value of $1500 \mathrm{~s} / \mathrm{mm}^{2}$ shows focal increased signal (arrow). C: $D_{\text {app }}$ map and $D: K_{\text {app }}$ map obtained using non-Gaussian kurtosis model show visually decreased $D_{\text {app }}$ and increased $\mathrm{K}_{\mathrm{app}}$ in lesion (arrow). E: Superimposed signal-intensity decay plots obtained using monoexponential fit for b-values up to $1000 \mathrm{~s} /$ $\mathrm{mm}^{2}$ (dashed red line) and kurtosis fit for b-values up to $2000 \mathrm{~s} / \mathrm{mm}^{2}$ (solid blue line) show improved fitting of the raw data using kurtosis fit given its capturing of the curvature that occurs at b-values beyond $1000 \mathrm{~s} / \mathrm{mm}^{2}$. Quantitative assessment reveals elevated $K_{a p p}$, reflecting this curvature, as well as discrepancy between $A D C_{0-1000}$ and $D_{\text {app }}$.

of tissue diffusion, given its correction for non-Gaussian diffusion behavior.

$\mathrm{K}_{\text {app }}$ (unitless) equals 0 in the setting of completely Gaussian diffusion. ${ }^{34}$ Biologic tissues tend to exhibit $\mathrm{K}_{\mathrm{app}}$ values between 0 and $1 .{ }^{26} \mathrm{~K}_{\text {app }}$ values greater than 1 indicate increased contributions attributable to kurtosis behavior. ${ }^{7,33}$ Studies also suggest lowering of $\mathrm{K}$ in the setting of posttreatment tumor necrosis. ${ }^{33,47}$ Postprocessing software commonly applies a maximal possible upper limit for $\mathrm{K}_{\mathrm{app}}$, above which the value is likely to represent an outlier due to motion, noise, or other artifact. ${ }^{26}$ While $K_{\text {app }}$ theoretically may be negative, it will measure at least 0 in the setting of water exchange between multiple compartments, as is generally the case within biologic tissues, ${ }^{7,26}$ and 0 can therefore be used as a minimal value for $K_{a p p}$ in curvefitting procedures.

Reductions in $\mathrm{D}_{\mathrm{app}}$ are commonly, although not always, associated with elevations in $\mathrm{K}_{\text {app }} \cdot{ }^{10,33,34}$ As a result, the $\mathrm{K}_{\text {app }}$ parametric map may visually appear analogous to an inverted version of the $\mathrm{D}_{\text {app }}$ parametric map, both indicating abnormal diffusion behavior in similar anatomic locations. Therefore, a simple visual assessment of the two maps may be diagnostically insufficient. Rather, quantitative analysis incorporating direct measurements of $\mathrm{D}_{\text {app }}$ and $\mathrm{K}_{\mathrm{app}}$ values is recommended in order to fully harness the complementary role of kurtosis in distinguishing tissue pathology (Figs. 3, 4). In some circumstances, such as viscous or turbid fluid, reduced $D_{\text {app }}$ may be encountered, without elevated $\mathrm{K}_{\mathrm{app}}$ (Fig. 5).

\section{Pitfalls}

First, it is suggested to avoid using a monoexponential fit for DWI acquisitions that include ultrahigh b-values over $1000 \mathrm{~s} /$ $\mathrm{mm}^{2}$. For such acquisitions, the monoexponential model no longer applies, and the obtained estimates of ADC will be less meaningful and potentially incorrect. In this context, it may be desired to both compute ADC maps with b-values less than $1000 \mathrm{~s} / \mathrm{mm}^{2}$, as well as have very high b-value signal intensity images available for interpretation. ${ }^{48}$ In this circumstance, the acquired ultrahigh b-value images should be excluded from the ADC map computation. Alternatively, two separate diffusion acquisitions may be performed altogether, one using b-values up to $1000 \mathrm{~s} / \mathrm{mm}^{2}$ and one using ultrahigh 


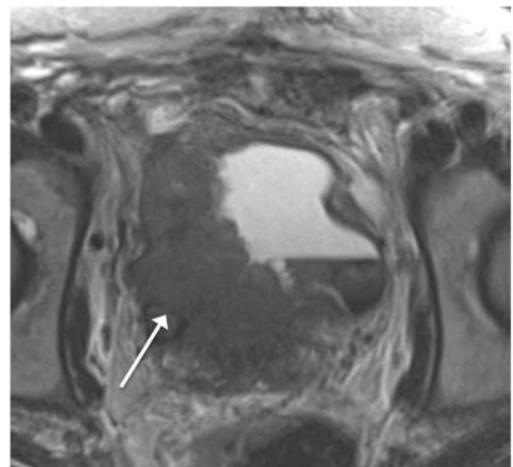

(a)

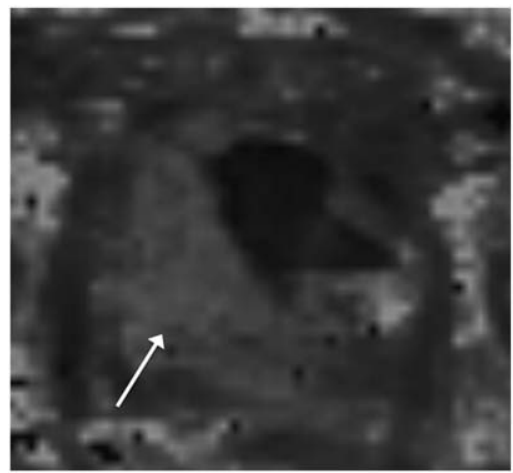

(d)

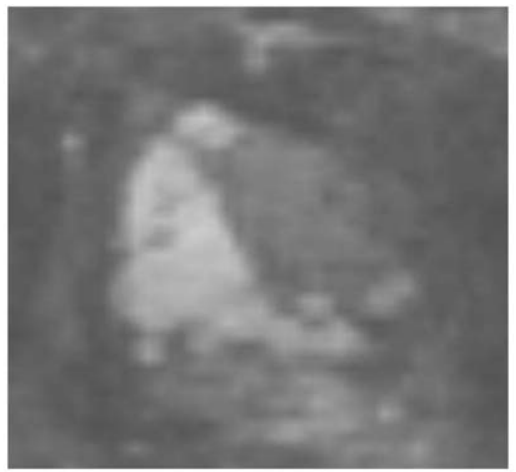

(b)

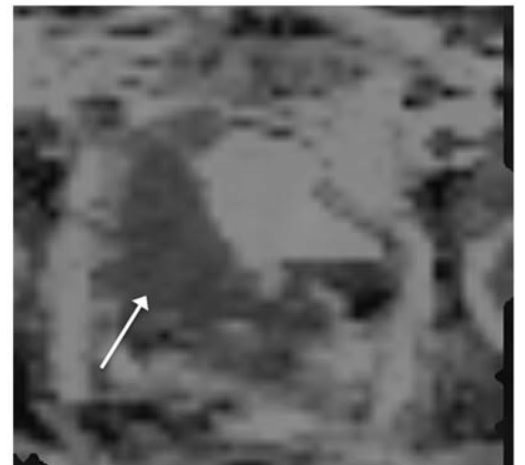

(c)

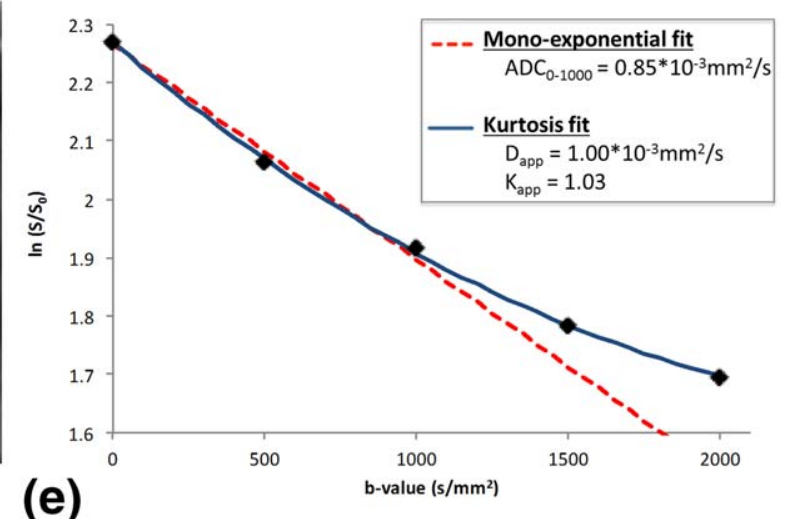

FIGURE 4: A 74-year-old man with high-grade urothelial carcinoma of the bladder. A: Axial $T_{2}$-weighted image shows large right posterolateral bladder mass (arrow) with gross extravesical extension. B: Diffusion-weighted image with ultra high b-value of $2000 \mathrm{~s} / \mathrm{mm}^{2}$ shows increased signal (arrow). C: $D_{\text {app }}$ map and $D$ : $K_{\text {app }}$ map obtained using non-Gaussian kurtosis model show visually decreased $D_{\text {app }}$ and increased $K_{\text {app }}$ in lesion (arrow). E: Superimposed signal-intensity decay plots obtained using monoexponential fit for b-values up to $1000 \mathrm{~s} / \mathrm{mm}^{2}$ (dashed red line) and kurtosis fit for b-values up to $2000 \mathrm{~s} / \mathrm{mm}^{2}$ (solid blue line) show improved fitting of the raw data using kurtosis fit given its capturing of the curvature that occurs at $b$-values beyond $1000 \mathrm{~s} / \mathrm{mm}^{2}$. Quantitative assessment reveals elevated $K_{a p p}$, reflecting this curvature, as well as discrepancy between $A D C_{0-1000}$ and $D_{a p p}$.

b-values, each optimized to achieve the maximal SNR possible for the b-value range used.

Likewise, the DKI model should not be applied when only b-values $<1000 \mathrm{~s} / \mathrm{mm}^{2}$ are acquired. ${ }^{33,36,44,49,50}$ Although software can mathematically fit the DKI equation using any range of b-values and output $\mathrm{D}_{\text {app }}$ and $\mathrm{K}_{\text {app }}$ parametric maps, there is no logical basis for doing so. Given the inclusion of an additional unknown variable in the DKI model, such postprocessing will provide a mathematically better goodness-of-fit to the raw data than a monoexponential model within the same b-value range. ${ }^{34}$ However, this improved goodness-of-fit should not be viewed as evidence of appropriateness, as the non-Gaussian diffusion behavior that underlies the DKI fit has minimal effect for b-values within this range. Since the curvature occurring at very high $b$-values is not present within this range, values of $D_{\text {app }}$ will tend to approach those of ADC. ${ }^{22,34}$ On this basis, radiologists should not characterize DKI fitting performed within only a standard b-value range as either representing or supporting the presence of non-Gaussian water behavior. Likewise, biomedical journals should require the appropriate use of higher b-values in studies that aim to explore the clinical utility of DKI. Finally, when comparing goodness-of-fit between different diffusion models, it is important to apply corrections to account for the different number of model parameters. $^{13}$

Third, the DKI model fits the diffusion signal decay well only when b-values do not exceed 2500-3000 s/ $\mathrm{mm}^{2}$ depending on $\mathrm{D}_{\text {app }}$ values. At higher b-values, the model, which is mathematical rather than physical, starts to fail, predicting increasing signal intensity as the b-value increases further, which is a physical non-sense. The b-value regime that we suggest for body applications of DKI is consistent with this upper limit.

Finally, as previously noted, radiologists need to ensure sufficient SNR on the high b-value images. This requirement represents an important barrier to widespread clinical application of DKI in body imaging at the present time. DKI postprocessing can technically be applied to SNR-poor image sets and yield $\mathrm{D}_{\text {app }}$ and $\mathrm{K}_{\text {app }}$ parametric maps from which mean values can be extracted. However, if the SNR is insufficient, then such data are prone to be heavily influenced by the image noise and thus may be spurious in nature. Therefore, the radiologist must always be aware of 


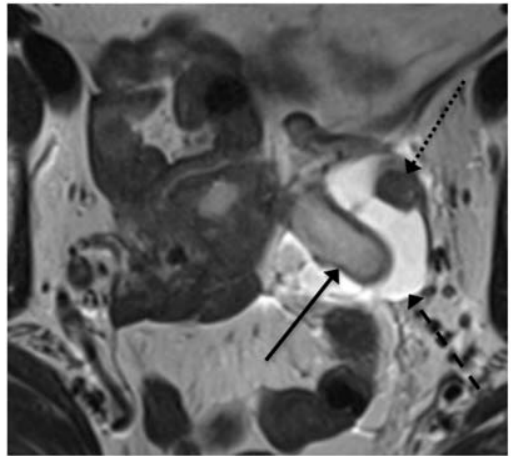

(a)

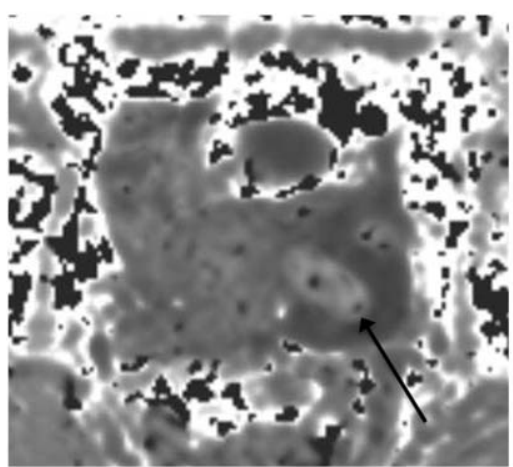

(d)

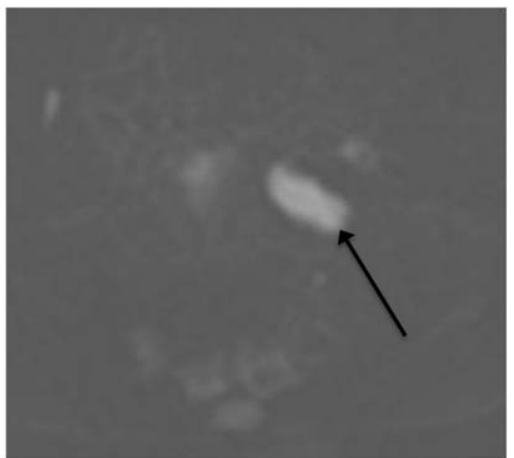

(b)

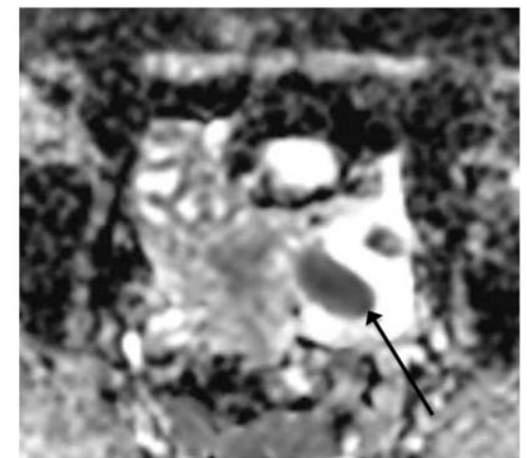

(c)

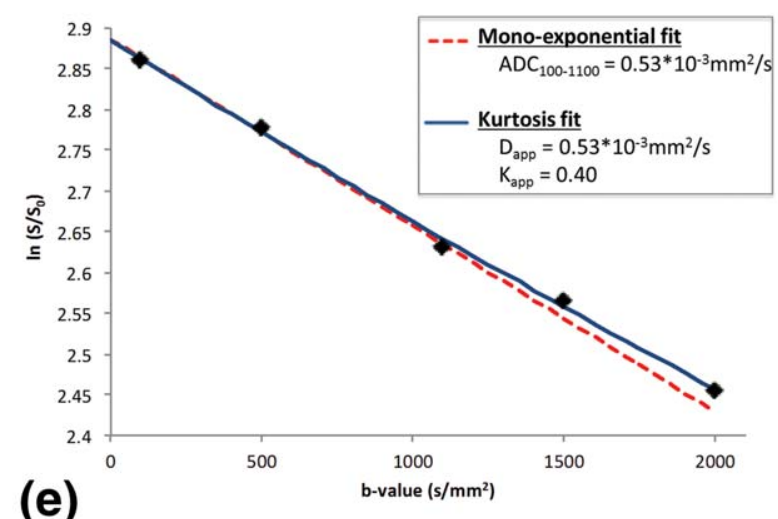

FIGURE 5: A 67-year-old woman with cystic adnexal lesion on ultrasound. MRI was performed for further evaluation. A: Axial $T_{2-}$ weighted image shows a tubular area of $T_{2}$-hyperintensity (solid arrow) representing a hydrosalpinx in middle of a peritoneal inclusion cyst (PIC, dashed arrow) that entraps the left ovary (dotted arrow), which can be seen along the left lateral wall of the PIC. B: Diffusion-weighted image with ultra high b-value of $2000 \mathrm{~s} / \mathrm{mm}^{2}$ shows marked increased signal in the hydrosalpinx (arrow). C: $D_{\text {app }}$ map and $D: K_{\text {app }}$ map obtained using DKI modeling show visually markedly decreased $D_{\text {app }}$ and intermediate $K_{a p p}$ in the tubular structure (arrow). E: Superimposed signal-intensity decay plots obtained using monoexponential fit for b-values up to $1100 \mathrm{~s} / \mathrm{mm}^{2}$ (dashed red line) and kurtosis fit for b-values up to $2000 \mathrm{~s} / \mathrm{mm}^{2}$ (solid blue line) show essentially identical fits without curvature at $b$-values beyond $1100 \mathrm{~s} / \mathrm{mm}^{2}$. The monoexponential decay of this tissue is consistent with its relatively acellular content. For this viscous fluid, quantitative DKI reveals markedly decreased $D_{a p p}$, without significant elevation in $K_{a p p}$ as well as essentially identical values for $A D C_{0-1000}$ and $D_{\text {app }}$.

SNR issues during data acquisition and postprocessing, and implement adjustments appropriately. One method of improving the accuracy of parameter estimates is to use averaged signal intensity values over a user-defined ROI as inputs to model fitting (as opposed to individual voxel fitting), which also enables confidence intervals of parameters estimates to be obtained, if required.

\section{Clinical Review}

In this section we summarize applications of DKI outside the brain that have been reported within the peer-reviewed literature (Table 3).

\section{Prostate}

A number of studies have evaluated the use of ultrahigh bvalue images in detection and characterization of prostate cancer. ${ }^{4,18,19,51}$ Indeed, the use of ultrahigh b-values for prostate cancer detection are incorporated into the PIRADS v. 2.0 guidelines, ${ }^{48}$ that state that ultrahigh b-value images should be routinely acquired and inspected for focal areas of hyperintensity, although such areas must also be secondarily evaluated on the ADC map (obtained with bvalues $\leq 1000 \mathrm{~s} / \mathrm{mm}^{2}$ ), dynamic contrast-enhanced MRI, and $T_{2}$-weighted imaging. These recommendations are corroborated in part from quantitative DKI studies conducted in the prostate, with at least 13 studies as of this writing measuring $\mathrm{K}_{\mathrm{app}}$ in benign or malignant prostate tissue, ${ }^{10,20,21,29,37,44,52-58}$ although not all used a b-value over $1000 \mathrm{~s} / \mathrm{mm}^{2}{ }^{44,52}$ DKI has shown incremental improvements compared with standard DWI for differentiating benign and malignant prostate tissue, ${ }^{37,54,55}$ as well as low and high grade tumors. ${ }^{10}$ However, not all studies have confirmed a significant diagnostic improvement in tumor grade differentiation using DKI. ${ }^{21,57}$

A number of investigations report improved diagnostic performance of DWI for prostate cancer detection when using b-values greater than $1000 \mathrm{~s} / \mathrm{mm}^{2}{ }^{4,14,30}$ While this improvement may relate to greater suppression of background benign tissue and thus higher lesion-to-background contrast at higher b-values, this effect only partly accounts 
Journal of Magnetic Resonance Imaging

TABLE 3. Summary of Existing Literature of DKI Outside the Brain

\begin{tabular}{|c|c|c|c|c|c|}
\hline $\begin{array}{l}\text { First } \\
\text { author }\end{array}$ & Year & $\begin{array}{l}\text { Maximal } \\
\text { b-value }^{\mathbf{a}}\end{array}$ & Organ & Pathology & Comment \\
\hline Quentin & 2012 & 800 & Prostate & Cancer & \\
\hline Rosenkrantz & 2012 & 2000 & Prostate & Cancer & \\
\hline Rosenkrantz & 2013 & 2000 & Prostate & Cancer & \\
\hline Bourne & 2014 & 2104 & Prostate & - & Ex vivo \\
\hline Mazzoni & 2014 & 2300 & Prostate & Cancer & \\
\hline Quentin & 2014 & 1000 & Prostate & Cancer & \\
\hline Suo & 2014 & 2000 & Prostate & Cancer & \\
\hline Tamura & 2014 & 1500 & Prostate & Cancer & \\
\hline Toivonen & 2014 & 2000 & Prostate & Cancer & \\
\hline Roethke & 2015 & 2000 & Prostate & Cancer & \\
\hline Jambor & 2015 & 2000 & Prostate & Cancer & \\
\hline Panagiotaki & 2015 & 3000 & Prostate & Cancer & \\
\hline Merisaari & 2015 & 2000 & Prostate & Cancer & \\
\hline Jansen & 2010 & 1500 & $\begin{array}{l}\text { Head and } \\
\text { neck }\end{array}$ & SCC & \\
\hline $\mathrm{Lu}$ & 2012 & 1448 & $\begin{array}{l}\text { Head and } \\
\text { neck }\end{array}$ & SCC nodal metastases & \\
\hline Yuan & 2014 & 1500 & $\begin{array}{l}\text { Head and } \\
\text { neck }\end{array}$ & Nasopharyngeal carcinoma & \\
\hline Chen & 2015 & 1500 & $\begin{array}{l}\text { Head and } \\
\text { neck }\end{array}$ & Nasopharyngeal carcinoma & \\
\hline Iima & 2014 & 2500 & Breast & Cancer; other benign lesions & \\
\hline Nogueira & 2014 & 3000 & Breast & Cancer; other benign lesions & \\
\hline Wu & 2014 & 2000 & Breast & Cancer; other benign lesions & \\
\hline Trampel & 2006 & 0.15 & Lung & Small airway disease & Used hyperpolarized ${ }^{3} \mathrm{He}$ \\
\hline Heusch & 2013 & 2000 & Lung & Nonsmall-cell lung cancer & $\begin{array}{l}\text { Part of }{ }^{18} \mathrm{~F}-\mathrm{FDG} \\
\text { PET/MRI }\end{array}$ \\
\hline Pentang & 2014 & 600 & Kidney & - & \\
\hline Huang & 2015 & 1000 & Kidney & - & \\
\hline Rosenkrantz & 2012 & 2000 & Liver & Hepatocellular carcinoma & $\begin{array}{l}\text { Ex vivo liver } \\
\text { explants }\end{array}$ \\
\hline Anderson & 2014 & 3500 & Liver & Fibrosis & $\begin{array}{l}\text { Ex vivo murine } \\
\text { specimens }\end{array}$ \\
\hline Goshima & 2015 & 2000 & Liver & Hepatocellular carcinoma & \\
\hline Suo & 2015 & 2000 & Bladder & Cancer & \\
\hline Marschar & 2015 & 5600 & Calf muscle & - & \\
\hline Lohezic & 2014 & 10000 & Myocardium & & $\begin{array}{l}\text { Ex vivo rat specimens; } \\
\text { Q-space imaging }\end{array}$ \\
\hline Yamada & 2015 & 7163 & Esophagus & Cancer & Ex vivo; Q-space imaging \\
\hline Filli & 2014 & 800 & Whole body & - & \\
\hline
\end{tabular}


for the benefit observed. The probing of the interactions between water and tissue elements that start to be seen only at very high b-values probably also contributes to improved lesion conspicuity. This distinction is important because bvalue images (of any magnitude) can alternatively be generated/synthesized/computed from images obtained at standard b-values. ${ }^{31,32}$ When such extrapolations are based on a standard monoexponential fit of the SI decay plot, nonGaussian water behavior manifesting at ultrahigh b-values cannot be truly ascertained.

\section{Head and Neck}

Three studies performed DKI using a b-value of $\sim 1500 \mathrm{~s} /$ $\mathrm{mm}^{2}$ for head and neck cancers, ${ }^{11,59,60}$ showing improved goodness-of-fit using DKI as well as generation of nonredundant information. An additional study using a b-value of $1500 \mathrm{~s} / \mathrm{mm} 2$ in patients undergoing neoadjuvant chemotherapy for nasopharyngeal carcinoma suggested DKI to be superior to standard monoexponential DWI for predicting early treatment response. ${ }^{61}$

\section{Breast}

Three studies have applied DKI using a maximal b-value of 2000-3000 s/ $\mathrm{mm}^{2}$ for assessment of breast diseases, ${ }^{12,62,63}$ demonstrating non-Gaussian diffusion behavior. Two of these studies reported strong performance of both $\mathrm{D}_{\text {app }}$ and $\mathrm{K}_{\text {app }}$ for differentiating benign and malignant breast tissue, although with conflicting results in terms of which of these was the better-performing metric. ${ }^{12,63}$ The remaining study reported that fibroadenomas and fibrocystic change showed alterations only in $\mathrm{K}_{\mathrm{app} .}{ }^{62}$

\section{Lung}

One study performed DKI of the lungs using hyperpolarized helium in a patient with small airway disease, showing an alteration in kurtosis, although not in ADC. ${ }^{64}$ Another study showed a significant correlation between $K_{\text {app }}$ and the standardized uptake value of nonsmall-cell lung cancer imaged by hybrid $18^{\mathrm{F}}$-FDG PET/MRI. ${ }^{65}$

\section{Kidney}

Two studies have attempted DKI of normal renal tissue, although using a maximal b-value of 600 or $1000 \mathrm{~s} /$ $\mathrm{mm}^{2}{ }^{49,50}$ Interestingly, one of these studies reported higher $\mathrm{K}_{\mathrm{app}}$ in the renal medulla, whereas the other reported higher $\mathrm{K}_{\text {app }}$ in the cortex. ${ }^{49,50}$ Nonetheless, the maximal b-values used in these studies are too low to support conclusions regarding the feasibility or utility of DKI in the kidneys.

\section{Liver}

One study explored DKI using a maximal b-value of $2000 \mathrm{~s} /$ $\mathrm{mm}^{2}$ in hypervascular hepatocellular carcinoma (HCC), reporting higher performance of $\mathrm{K}_{\mathrm{app}}$ than $\mathrm{ADC}$ for assessing posttreatment viability. ${ }^{47}$ In addition, in an ex vivo study of a murine liver specimens with known fibrosis, DKI exhibited better goodness-of-fit than a monoexponential model. ${ }^{13}$ Also, in an ex vivo study of HCC within human liver explants $\mathrm{K}_{\text {app }}$ was positively correlated with tumor cellularity and reduced $\mathrm{K}_{\text {app }}$ was observed in necrotic treated lesions. ${ }^{33}$

\section{Other}

Additional preliminary studies have demonstrated the feasibility of DKI within bladder (showing higher area under the curve of $K_{\text {app }}$ than ADC for differentiating low- and highgrade cancer), ${ }^{38}$ human calf muscle in vivo, ${ }^{66}$ rat myocardium ex vivo, ${ }^{67}$ and human esophageal carcinoma ex vivo. ${ }^{68}$ Another study demonstrated the feasibility of performing whole-body DKI processing in healthy human volunteers, although using a maximal b-value of $800 \mathrm{~s} / \mathrm{mm}^{2} .{ }^{36}$ At this $\mathrm{b}$-value, values of $\mathrm{D}_{\text {app }}$ will tend to approach those of ADC, and radiologists should not characterize non-Gaussian fitting performed within this standard b-value range as either representing or supporting DKI.

\section{Future Directions}

The potential of quantitative DKI, incorporating not just visual assessment of ultrahigh b-value images but also measuring $\mathrm{K}_{\mathrm{app}}$, remains to be fully explored. Therefore, for initial validation studies examining the role of ultrahigh b-value DWI, radiologists are advised to include a quantitative DKI evaluation if possible. In particular, we suggest that DKI be explored during drug development to potentially establish $\mathrm{K}_{\mathrm{app}}$ and $\mathrm{D}_{\text {app }}$ as pharmacodynamic biomarkers for treatments that would be expected to lead to decreased cellular complexity with treatment response, thereby leading to loss of kurtosis and increased diffusivity. ${ }^{33}$ In this context, the potential of $\mathrm{K}_{\text {app }}$ to serve as an independent measure of response beyond ADC can be explored. ${ }^{23,69}$ Also, quantitative DKI could become part of multiparametric imaging assessments, for instance to support radiomics development, to improve biologic understanding of tissue or tumor heterogeneity at multiple scales, to integrate with other existing biomarkers, and to support emerging clinical applications such as radiotherapy planning on the basis of quantitative tissue characteristics. ${ }^{70}$ Ultimately, to establish DKI metrics as potential biomarkers a robust and rigorous biomarker development pathway is required, including establishment of quality control, measurement repeatability/reproducibility, understanding of sources of measurement error, evaluation of test-retest and interplatform repeatability, comparison of DKI metrics with ADC in multicenter studies, and validation of changes in DKI metrics using histopathology, molecular pathology, and other laboratory biomarkers in preclinical and clinical settings.

In conclusion, in comparison to the role of standard DWI and ADC in assessing extracellular water diffusion, DKI probes non-Gaussian interactions of water molecules within tissue environments and, through more advanced 
mathematical curve fitting, provides an additional parameter, $K_{\text {app}}$, that presumably reflects the heterogeneity and irregularity of cellular microstructure, as well as the amount of interfaces within cellular tissues. Nonetheless, careful attention to detail with which body radiologists may not be currently familiar is important to ensure reliable results. While DKI is acquired using a standard DWI sequence, although incorporating ultrahigh b-values in order to detect the non-Gaussian behavior, additional dedicated postprocessing software is required for analysis and generation of DKI parametric maps. In this article we have suggested a maximal b-value of 1500-2000 s/m $\mathrm{mm}^{2}$ for DKI applications in the body, lower than typically used in the brain. In recent years, a small number of studies have explored the role of DKI outside the brain. The most investigated organ is the prostate gland, with preliminary studies suggesting potentially improved tumor detection and grading using DKI.

Currently, DKI is still largely a research tool, and few compelling data support its routine use over conventional DWI measurements in oncologic assessments. Nonetheless, the technique is at a stage in which it can be explored in wider clinical settings. A robust understanding of DKI is important for radiologists to better understand what $\mathrm{K}_{\mathrm{app}}$ and $\mathrm{D}_{\text {app }}$ mean in the context of different tumors and how these metrics vary between tumor types and in response to treatment. We therefore encourage body radiologists to employ DKI using the suggestions presented in this review, especially in the framework of future investigations of ultrahigh b-value imaging. Such efforts will enhance our knowledge of DKI findings and help better define the benefits and drawbacks of this novel technique.

\section{Acknowledgment}

We thank David J. Collins for review of the article. Dow$\mathrm{Mu}$ Koh is supported by the NIHR Biomedical Research Centre at the Royal Marsden Hospital and Institute of Cancer Research, UK.

\section{Conflict of Interest}

Anwar Padhani is on the speaker's bureau and has a research agreement with Siemens Healthcare. Thomas L. Chenevert is a coinventor of intellectual property assigned to and managed by the University of Michigan. No other authors have any conflicts to report.

\section{References}

1. Koh DM, Collins DJ. Diffusion-weighted MRI in the body: applications and challenges in oncology. AJR Am J Roentgenol 2007;188:1622-1635.

2. Padhani AR, Liu G, Koh DM, et al. Diffusion-weighted magnetic resonance imaging as a cancer biomarker: consensus and recommendations. Neoplasia 2009;11:102-125.

3. Le Bihan D. Apparent diffusion coefficient and beyond: what diffusion MR imaging can tell us about tissue structure. Radiology 2013;268:318-322.
4. Katahira K, Takahara T, Kwee TC, et al. Ultra-high-b-value diffusionweighted MR imaging for the detection of prostate cancer: evaluation in 201 cases with histopathological correlation. Eur Radiol 2011;21:188-196.

5. Raab P, Hattingen E, Franz K, Zanella FE, Lanfermann H. Cerebral gliomas: diffusional kurtosis imaging analysis of microstructural differences. Radiology 2010;254:876-881.

6. Chabert S, Mecca CC, Le Bihan DJ. Relevance of the information about the diffusion distribution in invo given by kurtosis in q-space imaging. In: Proc 12th Annual Meeting ISMRM, Kyoto; 2004. p 1238.

7. Jensen JH, Helpern JA, Ramani A, Lu H, Kaczynski K. Diffusional kurtosis imaging: the quantification of non-Gaussian water diffusion by means of magnetic resonance imaging. Magn Reson Med 2005;53: 1432-1440.

8. Wu EX, Cheung MM. MR diffusion kurtosis imaging for neural tissue characterization. NMR Biomed 2010;23:836-848.

9. Fieremans $E$, Jensen JH, Helpern JA. White matter characterization with diffusional kurtosis imaging. Neurolmage 2011;58:177-188.

10. Rosenkrantz AB, Sigmund EE, Johnson $G$, et al. Prostate cancer: feasibility and preliminary experience of a diffusional kurtosis model for detection and assessment of aggressiveness of peripheral zone cancer. Radiology 2012;264:126-135.

11. Jansen JF, Stambuk HE, Koutcher JA, Shukla-Dave A. Non-Gaussian analysis of diffusion-weighted MR imaging in head and neck squamous cell carcinoma: a feasibility study. AJNR Am J Neuroradiol 2010;31:741-748.

12. lima M, Yano K, Kataoka M, et al. Quantitative non-Gaussian diffusion and intravoxel incoherent motion magnetic resonance imaging: differentiation of malignant and benign breast lesions. Invest Radiol 2015; 50:205-211.

13. Anderson SW, Barry B, Soto J, Ozonoff A, O'Brien M, Jara H. Characterizing non-Gaussian, high b-value diffusion in liver fibrosis: stretched exponential and diffusional kurtosis modeling. J Magn Reson Imaging JMRI 2014;39:827-834.

14. Metens T, Miranda D, Absil J, Matos C. What is the optimal b value in diffusion-weighted MR imaging to depict prostate cancer at 3T? Eur Radiol 2012;22:703-709.

15. Vural M, Ertas G, Onay A, et al. Conspicuity of peripheral zone prostate cancer on computed diffusion-weighted imaging: comparison of cDWI1500, cDWI2000, and cDWI3000. BioMed Res Int 2014; 2014:768291.

16. Tamada $T$, Kanomata $N$, Sone $T$, et al. High b value $(2,000 \mathrm{~s} / \mathrm{mm} 2)$ diffusion-weighted magnetic resonance imaging in prostate cancer at 3 Tesla: comparison with 1,000 s/mm2 for tumor conspicuity and discrimination of aggressiveness. PLoS One 2014;9:e96619.

17. Ahn SJ, Choi SH, Kim YJ, et al. Histogram analysis of apparent diffusion coefficient map of standard and high B-value diffusion MR imaging in head and neck squamous cell carcinoma: a correlation study with histological grade. Acad Radiol 2012;19:1233-1240.

18. Kitajima K, Kaji Y, Kuroda K, Sugimura K. High b-value diffusionweighted imaging in normal and malignant peripheral zone tissue of the prostate: effect of signal-to-noise ratio. Magn Reson Med Sci 2008;7:93-99.

19. Kim CK, Park BK, Kim B. High-b-value diffusion-weighted imaging at $3 \mathrm{~T}$ to detect prostate cancer: comparisons between $\mathrm{b}$ values of 1,000 and 2,000 s/mm2. AJR Am J Roentgenol 2010;194:W33-37.

20. Bourne RM, Panagiotaki E, Bongers A, Sved P, Watson G, Alexander DC. Information theoretic ranking of four models of diffusion attenuation in fresh and fixed prostate tissue ex vivo. Magn Reson Med 2014;72:1418-1426.

21. Toivonen J, Merisaari H, Pesola M, et al. Mathematical models for diffusion-weighted imaging of prostate cancer using $b$ values up to $2000 \mathrm{~s} / \mathrm{mm}$ : Correlation with Gleason score and repeatability of region of interest analysis. Magn Reson Med 2014 [Epub ahead of print].

22. Grinberg F, Farrher E, Ciobanu L, Geffroy F, Le Bihan D, Shah NJ. Non-Gaussian diffusion imaging for enhanced contrast of brain tissue affected by ischemic stroke. PLoS One 2014;9:e89225. 
23. Padhani AR, Makris A, Gall P, Collins DJ, Tunariu N, de Bono JS. Therapy monitoring of skeletal metastases with whole-body diffusion MRI. J Magn Reson Imaging JMRI 2014;39:1049-1078.

24. Nonomura $\mathrm{Y}$, Yasumoto $\mathrm{M}$, Yoshimura $\mathrm{R}$, et al. Relationship between bone marrow cellularity and apparent diffusion coefficient. J Magn Reson Imaging JMRI 2001;13:757-760.

25. Le Bihan D. The 'wet mind': water and functional neuroimaging. Phys Med Biol 2007;52:R57-90.

26. Jensen JH, Helpern JA. MRI quantification of non-Gaussian water diffusion by kurtosis analysis. NMR Biomed 2010;23:698-710.

27. White NS, Dale AM. Distinct effects of nuclear volume fraction and cell diameter on high b-value diffusion MRI contrast in tumors. Magn Reson Med 2014:72:1435-1443.

28. Lawrence E, Goldman D, Gallagher F, et al. Evaluating the Relationship between Gleason Score, Tumor Tissue Composition, and Diffusion Kurtosis Imaging in Intermediate/High-risk Prostate Cancer Whole-mount Specimens. Radiological Society of North America 2014 Scientific Assembly and Annual Meeting, Chicago IL. http://archive. rsna.org/2014/14003112.html. Accessed April 26, 2015.

29. Panagiotaki E, Chan RW, Dikaios N, et al. Microstructural characterization of normal and malignant human prostate tissue with vascular, extracellular, and restricted diffusion for cytometry in tumours magnetic resonance imaging. Invest Radiol 2015;50:218-227.

30. Rosenkrantz $A B$, Hindman N, Lim RP, et al. Diffusion-weighted imaging of the prostate: comparison of b1000 and b2000 image sets for index lesion detection. J Magn Reson Imaging JMRI 2013;38:694-700.

31. Maas MC, Futterer JJ, Scheenen TW. Quantitative evaluation of computed high $\mathrm{B}$ value diffusion-weighted magnetic resonance imaging of the prostate. Invest Radiol 2013;48:779-786.

32. Ueno Y, Takahashi S, Kitajima K, et al. Computed diffusion-weighted imaging using 3-T magnetic resonance imaging for prostate cancer diagnosis. Eur Radiol 2013;23:3509-3516.

33. Rosenkrantz $A B$, Sigmund $E E$, Winnick $A$, et al. Assessment of hepatocellular carcinoma using apparent diffusion coefficient and diffusion kurtosis indices: preliminary experience in fresh liver explants. Magn Reson Imaging 2012;30:1534-1540.

34. Lu H, Jensen JH, Ramani A, Helpern JA. Three-dimensional characterization of non-Gaussian water diffusion in humans using diffusion kurtosis imaging. NMR Biomed 2006;19:236-247.

35. Assaf Y, Ben-Bashat D, Chapman J, et al. High b-value q-space analyzed diffusion-weighted MRI: application to multiple sclerosis. Magn Reson Med 2002;47:115-126.

36. Filli L, Wurnig M, Nanz D, Luechinger R, Kenkel D, Boss A. Wholebody diffusion kurtosis imaging: initial experience on non-Gaussian diffusion in various organs. Invest Radiol 2014;49:773-778.

37. Tamura $\mathrm{C}$, Shinmoto $\mathrm{H}$, Soga $\mathrm{S}$, et al. Diffusion kurtosis imaging study of prostate cancer: preliminary findings. J Magn Reson Imaging JMRI 2014;40:723-729.

38. Suo S, Chen X, Ji X, et al. Investigation of the non-Gaussian water diffusion properties in bladder cancer using diffusion kurtosis imaging: a preliminary study. J Comput Assist Tomogr 2015;39:281-285.

39. Koh DM, Collins DJ, Orton MR. Intravoxel incoherent motion in bod diffusion-weighted MRI: reality and challenges. AJR Am J Roentgenol 2011;196:1351-1361.

40. Pyatigorskaya N, Le Bihan D, Reynaud O, Ciobanu L. Relationship between the diffusion time and the diffusion MRI signal observed at 17.2 Tesla in the healthy rat brain cortex. Magn Reson Med 2014;72: 492-500.

41. Glenn GR, Tabesh A, Jensen JH. A simple noise correction scheme for diffusional kurtosis imaging. Magn Reson Imaging 2015;33:124-133.

42. Choi JS, Kim MJ, Chung YE, et al. Comparison of breathhold, navigator-triggered, and free-breathing diffusion-weighted MRI for focal hepatic lesions. J Magn Reson Imaging JMRI 2013;38:109-118.

43. Veraart J, Poot DH, Van Hecke W, et al. More accurate estimation of diffusion tensor parameters using diffusion kurtosis imaging. Magn Reson Med 2011;65:138-145.
44. Quentin M, Pentang G, Schimmoller $L$, et al. Feasibility of diffusional kurtosis tensor imaging in prostate MRI for the assessment of prostate cancer: preliminary results. Magn Reson Imaging 2014;32: 880-885.

45. Kyriazi S, Blackledge M, Collins DJ, Desouza NM. Optimising diffusion-weighted imaging in the abdomen and pelvis: comparison of image quality between monopolar and bipolar single-shot spinecho echo-planar sequences. Eur Radiol 2010;20:2422-2431.

46. Lewis S, Kamath A, Chatterji M, et al. Diffusion-weighted imaging of the liver in patients with chronic liver disease: comparison of monopolar and bipolar diffusion gradients for image quality and lesion detection. AJR Am J Roentgenol 2015;204:59-68

47. Goshima S, Kanematsu M, Noda $Y$, Kondo H, Watanabe H, Bae KT. Diffusion kurtosis imaging to assess response to treatment in hypervascular hepatocellular carcinoma. AJR Am J Roentgenol 2015; 204:W543-549.

48. American College of Radiology. Magnetic Resonance Prostate Imaging Reporting and Data System (MR PI-RADS). http://www.acr.org/ Quality-Safety/Resources/PIRADS Accessed December 29, 2014.

49. Pentang G, Lanzman RS, Heusch $P$, et al. Diffusion kurtosis imaging of the human kidney: a feasibility study. Magn Reson Imaging 2014;32: 413-420.

50. Huang $Y$, Chen $X$, Zhang $Z$, et al. MRI quantification of non-Gaussian water diffusion in normal human kidney: a diffusional kurtosis imaging study. NMR Biomed 2015;28:154-161.

51. Rosenkrantz $A B$, Kong $X$, Niver $B E$, et al. Prostate cancer: comparison of tumor visibility on trace diffusion-weighted images and the apparent diffusion coefficient map. AJR Am J Roentgenol 2011;196:123-129.

52. Quentin M, Blondin D, Klasen J, et al. Comparison of different mathematical models of diffusion-weighted prostate MR imaging Magn Reson Imaging 2012;30:1468-1474.

53. Rosenkrantz AB, Prabhu V, Sigmund EE, Babb JS, Deng FM, Taneja SS. Utility of diffusional kurtosis imaging as a marker of adverse pathologic outcomes among prostate cancer active surveillance candidates undergoing radical prostatectomy. AJR Am J Roentgenol 2013:201:840-846.

54. Mazzoni LN, Lucarini S, Chiti S, Busoni S, Gori C, Menchi I. Diffusionweighted signal models in healthy and cancerous peripheral prostate tissues: comparison of outcomes obtained at different b-values. J Magn Reson Imaging JMRI 2014;39:512-518.

55. Suo $\mathrm{S}$, Chen $\mathrm{X}, \mathrm{Wu} \mathrm{L}$, et al. Non-Gaussian water diffusion kurtosis imaging of prostate cancer. Magn Reson Imaging 2014;32:421-427.

56. Jambor I, Merisaari $\mathrm{H}$, Taimen $\mathrm{P}$, et al. Evaluation of different mathematical models for diffusion-weighted imaging of normal prostate and prostate cancer using high b-values: a repeatability study. Magn Reson Med 2015;73:1988-1998.

57. Roethke MC, Kuder TA, Kuru TH, et al. Evaluation of diffusion kurtosis imaging versus standard diffusion imaging for detection and grading of peripheral zone prostate cancer. Invest Radiol 2015 [Epub ahead of print].

58. Merisaari $H$, Jambor I. Optimization of b-value distribution for four mathematical models of prostate cancer diffusion-weighted imaging using b values up to $2000 \mathrm{~s} / \mathrm{mm}$ (2): Simulation and repeatability study. Magn Reson Med 2015;73:1954-1969.

59. Lu Y, Jansen JF, Mazaheri Y, Stambuk HE, Koutcher JA, Shukla-Dave A. Extension of the intravoxel incoherent motion model to nonGaussian diffusion in head and neck cancer. J Magn Reson Imaging JMRI 2012:36:1088-1096.

60. Yuan J, Yeung DK, Mok GS, et al. Non-Gaussian analysis of diffusion weighted imaging in head and neck at 3T: a pilot study in patients with nasopharyngeal carcinoma. PLoS One 2014;9:e87024.

61. Chen $Y$, Ren $W$, Zheng D, et al. Diffusion kurtosis imaging predicts neoadjuvant chemotherapy responses within 4 days in advanced nasopharyngeal carcinoma patients. J Magn Reson Imaging JMRI 2015 [Epub ahead of print]. 
62. Nogueira L, Brandao S, Matos E, et al. Application of the diffusion kurtosis model for the study of breast lesions. Eur Radiol 2014;24: 1197-1203.

63. Wu D, Li G, Zhang J, Chang S, Hu J, Dai Y. Characterization of Breast Tumors Using Diffusion Kurtosis Imaging (DKI). PLoS One 2014;9: e113240.

64. Trampel R, Jensen JH, Lee RF, Kamenetskiy I, McGuinness G, Johnson G. Diffusional kurtosis imaging in the lung using hyperpolarized 3He. Magn Reson Med 2006;56:733-737.

65. Heusch P, Kohler J, Wittsack HJ, et al. Hybrid [(1) (8)F]-FDG PET/MRI including non-Gaussian diffusion-weighted imaging (DWI): preliminary results in non-small cell lung cancer (NSCLC). Eur J Radiol 2013;82: 2055-2060.

66. Marschar AM, Kuder TA, Stieltjes B, Nagel AM, Bachert P, Laun FB. In vivo imaging of the time-dependent apparent diffusional kurtosis in the human calf muscle. J Magn Reson Imaging JMRI 2015;41:15811590.

67. Lohezic M, Teh I, Bollensdorff $C$, et al. Interrogation of living myocardium in multiple static deformation states with diffusion tensor and diffusion spectrum imaging. Prog Biophys Mol Biol 2014;115: 213-225.

68. Yamada I, Hikishima K, Miyasaka N, et al. Esophageal carcinoma: Evaluation with $\mathrm{q}$-space diffusion-weighted MR imaging ex vivo. Magn Reson Med 2015;73:2262-2273.

69. Li SP, Padhani AR. Tumor response assessments with diffusion and perfusion MRI. J Magn Reson Imaging JMRI 2012;35:745-763.

70. Padhani AR, Miles KA. Multiparametric imaging of tumor response to therapy. Radiology 2010;256:348-364. 